\title{
Influence of Ionospheric Irregularities on GNSS Remote Sensing
}

\author{
M. V. Tinin ${ }^{1,2}$ \\ ${ }^{1}$ Institute of Solar-Terrestrial Physics SD RAS, P.O. Box 4026, Irkutsk 664033, Russia \\ ${ }^{2}$ Irkutsk State University, 20 Gagarin Boulevard, Irkutsk 664003, Russia
}

Correspondence should be addressed to M. V. Tinin; mtinin@api.isu.ru

Received 16 October 2014; Accepted 18 March 2015

Academic Editor: Hiroyuki Hashiguchi

Copyright (C) 2015 M. V. Tinin. This is an open access article distributed under the Creative Commons Attribution License, which permits unrestricted use, distribution, and reproduction in any medium, provided the original work is properly cited.

\begin{abstract}
We have used numerical simulation to study the effects of ionospheric irregularities on accuracy of global navigation satellite system (GNSS) measurements, using ionosphere-free (in atmospheric research) and geometry-free (in ionospheric research) dualfrequency phase combinations. It is known that elimination of these effects from multifrequency GNSS measurements is handicapped by diffraction effects during signal propagation through turbulent ionospheric plasma with the inner scale being smaller than the Fresnel radius. We demonstrated the possibility of reducing the residual ionospheric error in dual-frequency GNSS remote sensing in ionosphere-free combination by Fresnel inversion. The inversion parameter, the distance to the virtual screen, may be selected from the minimum of amplitude fluctuations. This suggests the possibility of improving the accuracy of GNSS remote sensing in meteorology. In the study of ionospheric disturbances with the aid of geometry-free combination, the Fresnel inversion eliminates only the third-order error. To eliminate the random TEC component which, like the measured average TEC, is the first-order correction, we should use temporal filtering (averaging).
\end{abstract}

\section{Introduction}

At present, remote sensing methods relying on groundbased and low-orbit observations of signals from global navigation satellite systems (GNSS) such as GLONASS and GPS are finding ever-widening application in environmental research [1-8]. These methods allow remote diagnostics of wide sectors of overground space, which makes them attractive for use especially in the Arctic region. However, the spatially and temporally variable ionosphere significantly affects the accuracy and reliability of GNSS remote sensing. Therefore, it is necessary to study effects of ionospheric irregularities on GNSS measurements and to find possibilities of their elimination. Unfortunately, the ionospheric variability impedes elimination of ionospheric errors through modeling. Particularly, the ionospheric error reduction with the aid of the well-known Klobuchar model $[9,10]$ does not exceed 50\% [11]. A more precise approach makes use of the frequency dependence of ionospheric error. Here we will restrict our consideration to phase measurements as being more precise versus measurements of time delay of signal (code measurements). Let us have a phase path $\varphi(f)$ measured taking into account the whole number of wavelengths determined by methods for phase ambiguity resolution (see, e.g., [12]). Then the first geometrical optics approximation yields $[9,10]$

$$
\varphi(f)=D-\frac{40.3}{f^{2}} I_{1 g}(f)+\cdots .
$$

Here the pseudo distance $D$ comprises, besides the distance between a satellite and a ground-based observer, all other frequency-independent corrections (errors opposite in sign) to phase path (e.g., delay in the troposphere); consider

$$
I_{1 g}=\int_{z_{0}}^{z_{t}} N\left(z^{\prime}\right) d z^{\prime}
$$

is the electron density integral along a ground-basedreceiver-satellite line, which is called total electron content (TEC); $f$ is the radiation frequency. The first-order correction, the second term in (1) proportional to $f^{-2}$, describes ionospheric plasma effects on GNSS signal propagation, ignoring diffraction effects, geomagnetic field effect (secondorder correction), and ray bending (the third-order correction). Here we use a generally accepted (see, e.g., [13-15]) 
notation of the $i$-order correction for the term in observation equations (1) and below, which is proportional to $f^{-i-1}$.

GNSS remote sensing methods (see, e.g., [1-8]) rest on the relation of terms on the right-hand side of (1) with environmental parameters. The distance included into $D$ depends on the observation point. Therefore, if the observer is on the Earth's surface, motions of this surface can be seen in $D$ variations. On the other hand, the atmospheric phase delay of signal included into $D$ is determined by the refractive index which depends on atmospheric temperature and density and vapor pressure. Thus, $D$ measurements can provide parameters important for meteorology. Estimating the second term in (1) allows us to acquire data on ionospheric plasma disturbances from the spatial and temporal TEC behavior.

In order to find the pseudo distance $D$ and TEC $I_{1 g}$ from (1), we need to perform dual-frequency measurements. These measurements give the following system:

$$
\begin{aligned}
& \varphi_{1}=\varphi_{g}\left(f_{1}\right)=D-\frac{40.3 I_{1 g}}{f_{1}^{2}}, \\
& \varphi_{2}=\varphi_{g}\left(f_{2}\right)=D-\frac{40.3 I_{1 g}}{f_{2}^{2}} .
\end{aligned}
$$

By solving system (3) for two unknowns $D$ and $I_{1 g}$, we obtain the result of the dual-frequency measurements:

$$
\begin{gathered}
D \approx D^{(2)}=\frac{\varphi_{1} f_{1}^{2}-\varphi_{2} f_{2}^{2}}{f_{1}^{2}-f_{2}^{2}}, \\
I^{(2)}=\frac{f_{1}^{2} f_{2}^{2}}{40.3} \frac{\varphi_{1}-\varphi_{2}}{f_{1}^{2}-f_{2}^{2}} .
\end{gathered}
$$

Equations (4) and (5) are so-called ionosphere-free and geometry-free observation combinations, respectively [16].

The dual-frequency measurements from formula (4) reduce the ionospheric distance-measurement error by $99 \%$ [17]. In practice, this is usually quite sufficient. However, in atmospheric investigations, in studies of crust movements during earthquakes and volcanic activity and other precisional GNSS measurements, this decimetric accuracy is not sufficient. Therefore, many researchers have been exploring the possibility of improving the accuracy of dual-frequency measurements.

The second-order correction which takes into account geomagnetic effects on the ionospheric refractive index is ignored in dual-frequency measurements (3). This correction has been examined in many studies (see, e.g., [1, 13-15, 1821]). It has been revealed that in the thin layer approximation whose error is $1-2 \mathrm{~mm}$ this correction is expressed through the first-order one $[13,15,22]$. Therefore, it can be taken into account by calculating with the familiar geomagnetic field model $[1,13-15]$ or by considering directly in dual-frequency measurements through modification of the ionosphere-free combination $[22,23]$.

After accounting for the second-order residual error, further accuracy improvement is associated with the consideration of the third-order error. In the geometrical optics approximation, this error is largely attributed to the path deviation from the straight line and can be eliminated in triple-frequency measurements [23, 24]. Yet, like dualfrequency measurements, triple-frequency measurements yield a system of three observation equations from which we can find $D$, first-order correction (TEC), and thirdorder correction (ray bending). This can only be done, however, when scales of ionospheric irregularities exceed the Fresnel radius, and the geometrical optics approximation is applicable. Unfortunately, scales of ionospheric irregularities range widely from tens of meters to tens of kilometers [2527]. In this case, the lower bound (so-called inner scale) of the ionospheric turbulent spectrum may be less than the Fresnel radius which varies with the elevation angle in the 200$700 \mathrm{~m}$ range (see, e.g., [23]). As a result, in triple-frequency measurements, as compared to dual-frequency ones, only the average error decreases, whereas the random error variance increases $[23,28]$.

The above results of studies of GNSS ionospheric errors largely refer to the results obtained with ionosphere-free combination (4), that is, during GNSS measurements of frequency-independent characteristics such as crust movement and tropospheric parameters. At present, however, GNSS measurements are widely used for studying traveling ionospheric disturbances as well as the ionosphere's behavior during earthquakes and magnetic storms [7, 29]. Yet TEC measurements (2) obtained from geometry-free combination (5) are utilized. It is obvious that in the study of this large-scale structure of the ionosphere and its dynamics, a significant role in formation of the measurement error will be played by ionospheric irregularities of less scale. In this paper, therefore, we will present results of investigations into residual ionospheric errors of dual-frequency GNSS measurements for both combinations (4) and (5).

\section{Ionospheric Effects in Single-Frequency GNSS Measurements}

Consider the behavior of GNSS signal in the form of harmonic wave with the time dependence $U(\mathbf{r}) \exp \{-i \omega t\}$. Since the GNSS satellite is far from the ionosphere, our task reduces to the study of incidence of the plane-wave $U_{0}(z)=$ $A_{0} \exp \left\{i k\left(z-z_{0}\right)\right\}$ with $k=2 \pi / \lambda$ ( $\lambda$ is the wavelength) on an inhomogeneous anisotropic ionosphere. Let the $z$-axis of our Cartesian coordinate system $\{x, y, z\}=\{\boldsymbol{\rho}, z\}$ pass through the source point $\mathbf{r}_{0}=\left\{0, z_{0}\right\}$ and the point $\left\{0, z_{t}\right\}$ located in the vicinity of the receiving point $\mathbf{r}_{t}=\left\{\boldsymbol{\rho}, z_{t}\right\}$. At GNSS frequencies, sizes of ionospheric irregularities $l_{N}$ usually exceed $\lambda$

$$
k l_{N}=\frac{2 \pi l_{N}}{\lambda} \gg 1 .
$$

With condition (6) fulfilled, when solving the wave equation, we use the second Rytov approximation and obtain the following solution $[30,31]$ :

$$
U(\mathbf{r})=U_{0}(z) \exp \left\{\Phi_{1}(\mathbf{r})+\Phi_{2}(\mathbf{r})\right\}
$$


where

$$
\Phi(\mathbf{r})=\Phi_{1}(\mathbf{r})+\Phi_{2}(\mathbf{r})
$$

is the complex phase whose first $\Phi_{1}(\mathbf{r})$ and second $\Phi_{2}(\mathbf{r})$ approximations have the forms:

$$
\begin{aligned}
\Phi_{1}(\mathbf{r})= & \frac{-i k 40.3}{f^{2}} \int_{z_{0}}^{z_{t}} d z^{\prime} \int_{-\infty}^{\infty} \int_{-\infty}^{\infty} d^{2} \kappa N_{\kappa}\left(\boldsymbol{\kappa}, z^{\prime}\right) \\
& \cdot \exp \left\{-\frac{i \kappa^{2}}{2} \frac{\left(z_{t}-z^{\prime}\right)}{k}+i \boldsymbol{\kappa} \boldsymbol{\rho}\right\}, \\
\Phi_{2}(\mathbf{r})= & \frac{i k}{2}\left(\frac{40.3}{f^{2}}\right)^{2} \int_{z_{0}}^{z_{t}} d z^{\prime} \\
& \cdot \int_{z_{0}}^{z^{\prime}} \int_{z_{0}}^{z^{\prime}} d z_{1} d z_{2} \int_{-\infty}^{\infty} \int_{-\infty}^{\infty} d^{2} \kappa_{1} \\
& \cdot \int_{-\infty}^{\infty} \int_{-\infty}^{\infty} d^{2} \kappa_{2} \boldsymbol{\kappa}_{1} \boldsymbol{\kappa}_{2} N_{\kappa}\left(\boldsymbol{\kappa}_{1}, z_{1}\right) N_{\kappa}\left(\boldsymbol{\kappa}_{2}, z_{2}\right) \\
& \cdot \exp \left\{-i S_{t}\left(\boldsymbol{\kappa}_{1}, \boldsymbol{\kappa}_{2}, z^{\prime}, z_{1}, z_{2}, k\right)+i\left(\boldsymbol{\kappa}_{1}+\boldsymbol{\kappa}_{2}\right) \boldsymbol{\rho}\right\} .
\end{aligned}
$$

Because the geomagnetic field effect on the velocity of GNSS signal propagation in the ionosphere can be taken into account, say, in the modified dual-frequency reception $[22$, 23], we do not discuss it here. In (9)

$$
N_{\kappa}(\boldsymbol{\kappa}, z)=\frac{1}{(2 \pi)^{2}} \int_{-\infty}^{\infty} \int_{-\infty}^{\infty} d^{2} \rho N(\boldsymbol{\rho}, z) \exp \{-i \kappa \boldsymbol{\rho}\}
$$

is the $2 \mathrm{D}$ spectrum of electron density of ionospheric plasma and

$$
\begin{aligned}
S_{t}\left(\boldsymbol{\kappa}_{1}, \boldsymbol{\kappa}_{2}, z^{\prime}, z_{1}, z_{2}, k\right) \\
\quad=\frac{\left[0.5 \kappa_{1}^{2}\left(z_{t}-z_{1}\right)+0.5 \kappa_{2}^{2}\left(z_{t}-z_{2}\right)+\boldsymbol{\kappa}_{1} \boldsymbol{\kappa}_{2}\left(z_{t}-z^{\prime}\right)\right]}{k} .
\end{aligned}
$$

The imaginary part of the complex phase $\Phi(\mathbf{r})$ determines the GNSS signal phase which, with the point of observation on the $z$ axis $(\rho=0)$, is

$$
\left.\operatorname{Im} \Phi(\mathbf{r})\right|_{\rho=0}+k\left(z-z_{0}\right)=k \varphi(\mathbf{r}) .
$$

The real part of the complex phase $\Phi(\mathbf{r})$ is associated with the level

$$
\left.\operatorname{Re} \Phi(\mathbf{r})\right|_{\rho=0}=\ln \left[\frac{A(\mathbf{r})}{A_{0}}\right] .
$$

Equations (12), (8), and (9) for the phase path $\varphi(\mathbf{r})$ yield the observation equation [31]:

$$
\varphi(f)=D-\frac{40.3}{f^{2}} I_{1 d}(f)-\left(\frac{40.3}{f^{2}}\right)^{2} I_{3 d}(f),
$$

where

$$
\begin{aligned}
I_{1 d}(f)= & \int_{z_{0}}^{z_{t}} d z^{\prime} \int_{-\infty}^{\infty} \int_{-\infty}^{\infty} d^{2} \kappa N_{\kappa}\left(\boldsymbol{\kappa}, z^{\prime}\right) \\
& \cdot \cos \left\{\frac{\kappa^{2}}{2} \frac{\left(z_{t}-z^{\prime}\right)}{k}\right\}, \\
I_{3 d}(f)= & -0.5 \int_{z_{0}}^{z} d z^{\prime} \int_{z_{0}}^{z^{\prime}} \int_{z_{0}}^{z^{\prime}} d z_{1} d z_{2} \\
& \cdot \int_{-\infty}^{\infty} \int_{-\infty}^{\infty} d^{2} \kappa_{1} \int_{-\infty}^{\infty} \int_{-\infty}^{\infty} d^{2} \kappa_{2} \kappa_{1} \kappa_{2} \\
& \times N_{\kappa}\left(\boldsymbol{\kappa}_{1}, z_{1}\right) N_{\kappa}\left(\boldsymbol{\kappa}_{2}, z_{2}\right) \\
& \cdot \cos \left\{S\left(\boldsymbol{\kappa}_{1}, \boldsymbol{\kappa}_{2}, z^{\prime}, z_{1}, z_{2}\right)\right\} .
\end{aligned}
$$

The second term on the right-hand side in (14) is the firstorder correction which, unlike (2), accounts for diffraction effects; the third term is the diffraction variant of the thirdorder correction. When the minimum size of irregularities $l_{m}=1 / \max (\kappa)$ exceeds the Fresnel radius $r_{\mathrm{Fr}}$

$$
l_{m}^{2} \gg r_{\mathrm{Fr}}^{2} \equiv \frac{\left(z_{t}-z^{\prime}\right)}{k},
$$

(14) and (15) give the geometrical optics approximation [23, 31]

$$
\varphi_{g}(f)=D^{(1)} \approx D-\frac{40.3 I_{1 g}}{f^{2}}-(40.3)^{2} f^{-4} I_{3 g},
$$

where

$$
I_{3 g}=0.5 \int_{z_{0}}^{z_{t}}\left[\left.\int_{z_{0}}^{z} \frac{\partial N\left(\boldsymbol{\rho}, z^{\prime}\right)}{\partial \boldsymbol{\rho}}\right|_{\boldsymbol{\rho}=0} d z^{\prime}\right]^{2} d z .
$$

In this case, unlike first approximation formula (1), besides the first-order correction proportional to the total electron content $I_{1 g}=\int_{z_{0}}^{z_{t}} N\left(z^{\prime}\right) d z^{\prime}$, the third-order correction (the final term on the right-hand side in (17)) associated with the path deviation from the straight line in the inhomogeneous ionosphere is taken into consideration. When ignoring the third-order correction, (17) yields (1) from which follow formulas (4) and (5) of dual-frequency measurements.

Thus, the errors to be considered are associated in the geometrical optics approximation with the GNSS ray bending. Moreover, it is necessary to correctly describe the interaction of the wave field with the irregularities for which conditions of the geometrical optics approximation (16) are not fulfilled. Expression (14), derived in the second Rytov approximation, accounts both for the ray bending and for the diffraction effects.

The electron density of ionospheric plasma is normally represented as sum of two components

$$
N(\mathbf{r})=\bar{N}(\mathbf{r})+\widetilde{N}(\mathbf{r}) .
$$


Here $\bar{N}(\mathbf{r})$ is the average electron density, that is, density of the background ionosphere and its medium-scale disturbances being of the utmost interest for ionosphere researchers [7, 29]; $\widetilde{N}(\mathbf{r})$ is the random component of electron density associated with small-scale irregularities.

The electron density $\bar{N}(\mathbf{r})$ of the large-scale background ionosphere and its medium-scale disturbances largely fulfills the condition of validity of the geometrical optics approximation (16) at GNSS frequencies. Besides, ray bending effects in the background ionosphere are sufficiently small $[13,24$, 31]. Therefore, diffraction effects in GNSS measurements are associated first and foremost with random small-scale irregularities caused by ionospheric plasma turbulence.

Random ionospheric irregularities are usually represented by a random, quasi-homogeneous, normally distributed field with a given spectrum $\Phi_{N}(\boldsymbol{\kappa}, \mathbf{r})$ related to the correlation function $\Psi_{N}(\Delta \mathbf{r}, \mathbf{r})=\langle\widetilde{N}(\mathbf{r}) \widetilde{N}(\mathbf{r}+\Delta \mathbf{r})\rangle$ by the relation

$$
\begin{aligned}
\Phi_{N}(\boldsymbol{\kappa}, \mathbf{r})= & (2 \pi)^{-3} \int_{-\infty}^{\infty} \int_{-\infty}^{\infty} \int_{-\infty}^{\infty} d^{3} \Delta r \Psi_{N}(\Delta \mathbf{r}, \mathbf{r}) \\
& \cdot \exp \{-i \boldsymbol{\kappa} \Delta \mathbf{r}\} .
\end{aligned}
$$

The spectrum of turbulence irregularities is often as follows [32]:

$$
\Phi_{N}(\kappa, z)=\frac{\sigma_{N}^{2}(z) \Gamma(p / 2) \kappa_{0}^{p-3}}{\pi^{3 / 2} \Gamma((p-3) / 2)}\left(\kappa_{0}^{2}+\kappa^{2}\right)^{-p / 2} \exp \left[-\frac{\kappa^{2}}{\kappa_{m}^{2}}\right],
$$

where $\sigma_{N}^{2}(z)$ is the dispersion of electron density fluctuations, $\kappa_{0}=2 \pi / L_{0}, \kappa_{m}=2 \pi / l_{m}$.

Spectrum (21) is bounded by the outer scale $L_{0}$ in the order of several tens of kilometers and by the inner scale $l_{m} \approx 50-700 \mathrm{~m}$.

By substituting (19) in (15), we can express characteristics of the random $I_{i d}(f)(i=1,3)$ through corresponding characteristics of the random field $N(\mathbf{r})$. For spectrum (21) the average $\bar{I}_{i d}(f)=\left\langle I_{i d}(f)\right\rangle$ and the correlation functions $\Psi_{i d}\left(f_{1}, f_{2}\right)=\left\langle I_{i d}\left(f_{1}\right) I_{i d}\left(f_{2}\right)\right\rangle$ are determined by the following relations [31]:

$$
\begin{gathered}
I_{0}=\left\langle I_{1 g}\right\rangle=\int_{z_{0}}^{z_{t}} \bar{N}\left(z^{\prime}\right) d z^{\prime}, \\
\bar{I}_{3 d}(f)=\left\langle I_{3 d}\right\rangle=k \frac{\sqrt{\pi} \Gamma(2-p / 2) \Gamma(p / 2) \kappa_{0}^{p-3}}{(p / 2-1) \Gamma((p-3) / 2) \kappa_{m}^{p-2}} \\
\cdot \int_{z_{0}}^{z_{t}} d z^{\prime} \sigma_{N}^{2}\left(z^{\prime}\right) \operatorname{Im} Q^{p / 2-1}\left(z^{\prime}, k_{1}\right),
\end{gathered}
$$

where $Q\left(z^{\prime}, k\right)=1-i \kappa_{m}^{2}\left(z_{t}-z^{\prime}\right) / k$,

$$
\begin{gathered}
\Psi_{1 d}\left(f_{1}, f_{2}\right) \\
=\left\langle\widetilde{I}_{1 d}\left(f_{1}\right) \widetilde{I}_{1 d}\left(f_{2}\right)\right\rangle \\
=\frac{2 \sqrt{\pi} \Gamma[(p-2) / 2]}{\kappa_{0} \Gamma[(p-3) / 2]} \int_{z_{0}}^{z_{t}} \sigma_{N}^{2}(z) d z \\
\cdot\left\{1+\left(\frac{\kappa_{0}}{\kappa_{m}}\right)^{p-2} \times \frac{\pi[\Gamma[(p-2) / 2]]^{-1}}{2 \sin (\pi p / 2)}\right. \\
\left.\cdot \operatorname{Re}\left[Q^{p / 2-1}\left(z, k_{+}\right)+Q^{p / 2-1}\left(z, k_{-}\right)\right]\right\} \\
k_{+,-}^{-1}=0.5\left(k_{1}^{-1} \pm k_{2}^{-1}\right), \\
\Psi_{3 d}\left(f_{1}, f_{2}\right)=\left\langle\widetilde{I}_{3 d}\left(f_{1}\right) \widetilde{I}_{3 d}\left(f_{2}\right)\right\rangle \\
=\Psi_{3 B}\left(f_{1}, f_{2}\right)+\Psi_{3 R}\left(f_{1}, f_{2}\right), \\
\Psi_{3 B}\left(f_{1}, f_{2}\right)=\frac{\sqrt{\pi} \Gamma(p / 2) \Gamma(2-p / 2) \kappa_{0}^{p-3}}{\Gamma((p-3) / 2) \kappa_{m}^{p-4}} \\
\cdot \int_{z_{0}}^{z_{t}} d z^{\prime} \int_{z_{0}}^{z^{\prime}} d z^{\prime \prime} \overline{\mathbf{p}}_{N}\left(z^{\prime}\right) \overline{\mathbf{p}}_{N}\left(z^{\prime \prime}\right) \\
\times \int_{z_{0}}^{z^{\prime \prime}} d z_{1} \sigma_{N}^{2}\left(z_{1}\right) \\
\cdot \operatorname{Re}\left[Q^{p / 2-2}\left(z_{1}, k_{+}\right)+Q^{p / 2-2}\left(z_{1}, k_{-}\right)\right],
\end{gathered}
$$

$$
\begin{aligned}
& \Psi_{3 R}\left(f_{1}, f_{2}\right) \\
& =\left[\frac{\Gamma(p / 2) \kappa_{0}^{p-3}}{\Gamma((p-3) / 2) \kappa_{m}^{p-4}}\right]^{2} 2 \Gamma(4-p) \\
& \quad \times \int_{z_{0}}^{z_{t}} d z^{\prime} \int_{z_{0}}^{z^{\prime}} d z^{\prime \prime} \int_{z_{0}}^{z^{\prime \prime}} d z_{1} \sigma_{N}^{2}\left(z_{1}\right) \\
& \quad \cdot \int_{z_{0}}^{z^{\prime \prime}} d z_{2} \sigma_{N}^{2}\left(z_{2}\right) \int_{-\pi}^{\pi} d \varphi_{-} \cos ^{2} \varphi_{-} \int_{0}^{\pi} d \varphi_{+} \sin ^{3-p} \varphi_{+} \\
& \quad \times \operatorname{Re}\left[V_{+}^{p-4}\left(f_{1}, f_{2}, z^{\prime}, z^{\prime \prime}\right)+V_{-}^{p-4}\left(f_{1}, f_{2}, z^{\prime}, z^{\prime \prime}\right)\right. \\
& \left.\quad+V_{+}^{p-4}\left(f_{1}, f_{2}, z^{\prime \prime}, z^{\prime}\right)+V_{-}^{p-4}\left(f_{1}, f_{2}, z^{\prime \prime}, z^{\prime}\right)\right],
\end{aligned}
$$

$$
\begin{aligned}
V_{+,-} & \left(f_{1}, f_{2}, z^{\prime}, z^{\prime \prime}\right) \\
= & 2 \sqrt{Q\left(z_{1}, k_{+,-}\right) Q\left(z_{2}, k_{+,-}\right)} \\
& -i \cos \varphi_{-} \sin \varphi_{+} \kappa_{m}^{2}\left[\frac{\left(z_{t}-z^{\prime}\right)}{k_{1}} \pm \frac{\left(z_{t}-z^{\prime \prime}\right)}{k_{2}}\right] .
\end{aligned}
$$


Note that contrary to the second term, the first term on the right-hand side in (25) accounts for the regular-refraction influence on the random component of the third-order error. According to the numerical simulation, the contribution of the first term is much less than that of the second term [31].

\section{Ionospheric Effects in Dual-Frequency GNSS Measurements}

After substituting (14) in dual-frequency ionosphere-free combination (4), we obtain

$$
D_{d}^{(2)}\left(f_{1}, f_{2}\right) \approx D-\Delta D_{1 d}^{(2)}\left(f_{1}, f_{2}\right)-\Delta D_{3 d}^{(2)}\left(f_{1}, f_{2}\right),
$$

where

$$
\Delta D_{1 d}^{(2)}\left(f_{1}, f_{2}\right)=\frac{40.3\left[I_{1 d}\left(f_{1}\right)-I_{1 d}\left(f_{2}\right)\right]}{\left(f_{1}^{2}-f_{2}^{2}\right)}
$$

is the first-order correction and

$$
\Delta D_{3 d}^{(2)}\left(f_{1}, f_{2}\right)=\frac{(40.3)^{2}\left[I_{3 d}\left(f_{1}\right) f_{1}^{-2}-I_{3 d}\left(f_{2}\right) f_{2}^{-2}\right]}{\left(f_{1}^{2}-f_{2}^{2}\right)}
$$

is the third-order correction.

By substituting (14) in geometry-free combination (5), we get

$$
I_{d}^{(2)}\left(f_{1}, f_{2}\right)=I_{0}-\Delta I_{1 d}^{(2)}\left(f_{1}, f_{2}\right)-\Delta I_{3 d}^{(2)}\left(f_{1}, f_{2}\right),
$$

where $I_{0}$ is, according to (22), the mean first-order TEC value,

$$
\Delta I_{1 d}^{(2)}\left(f_{1}, f_{2}\right)=\frac{\left[\widetilde{I}_{1 d}\left(f_{1}\right) f_{2}^{2}-\widetilde{I}_{1 d}\left(f_{2}\right) f_{1}^{2}\right]}{\left(f_{1}^{2}-f_{2}^{2}\right)}
$$

is the random component of the first-order TEC, and

$$
\Delta I_{3 d}^{(2)}\left(f_{1}, f_{2}\right)=\frac{40.3\left[I_{3 d}\left(f_{1}\right)\left(f_{2} / f_{1}\right)^{2}-I_{3 d}\left(f_{2}\right)\left(f_{1} / f_{2}\right)^{2}\right]}{\left(f_{1}^{2}-f_{2}^{2}\right)}
$$

is the third-order TEC correction.

Note that in (32), unlike (29), both the measured mean TEC $I_{0}$ and its random component $\Delta I_{1 d}^{(2)}\left(f_{1}, f_{2}\right)$ are firstorder corrections.

Now it is easy to find statistical characteristics of dualfrequency measurement errors. For measurements with ionosphere-free combination (4), the average correction is equal to the average third-order correction [23]:

$$
\begin{aligned}
\left\langle\Delta D_{d}^{(2)}\left(f_{1}, f_{2}\right)\right\rangle & \approx\left\langle\Delta D_{3 d}^{(2)}\left(f_{1}, f_{2}\right)\right\rangle \\
& =-\frac{(40.3)^{2}\left[\bar{I}_{3 d}\left(f_{1}\right) f_{1}^{-2}-\bar{I}_{3 d}\left(f_{2}\right) f_{2}^{-2}\right]}{\left(f_{1}^{2}-f_{2}^{2}\right)},
\end{aligned}
$$

where the average $\bar{I}_{3 R}(f)$ is determined by (23). The correction variance of this combination is [23]

$$
\begin{aligned}
\left\langle\left(\Delta \widetilde{D}_{d}^{(2)}\left(f_{1}, f_{2}\right)\right)^{2}\right\rangle= & \left\langle\left(\Delta \widetilde{D}_{1 d}^{(2)}\left(f_{1}, f_{2}\right)\right)^{2}\right\rangle \\
& +\left\langle\left(\Delta \widetilde{D}_{3 d}^{(2)}\left(f_{1}, f_{2}\right)\right)^{2}\right\rangle,
\end{aligned}
$$

where the first-order correction variance is

$$
\begin{aligned}
\langle( & \left.\left.\Delta \widetilde{D}_{1 d}^{(2)}\left(f_{1}, f_{2}\right)\right)^{2}\right\rangle \\
= & {\left[\frac{40.3}{\left(f_{1}^{2}-f_{2}^{2}\right)}\right]^{2} } \\
& \cdot\left[\Psi_{1 d}\left(f_{1}, f_{1}\right)+\Psi_{1 d}\left(f_{2}, f_{2}\right)-2 \Psi_{1 d}\left(f_{1}, f_{2}\right)\right]
\end{aligned}
$$

and the third-order correction variance is

$$
\begin{aligned}
\left\langle\left(\Delta \widetilde{D}_{3 d}^{(2)}\left(f_{1}, f_{2}\right)\right)^{2}\right\rangle= & {\left[\frac{(40.3)^{2}}{\left(f_{1}^{2}-f_{2}^{2}\right)}\right]^{2} } \\
& \times\left[f_{1}^{-4} \Psi_{3 d}\left(f_{1}, f_{1}\right)+f_{2}^{-4} \Psi_{3 d}\left(f_{2}, f_{2}\right)\right. \\
& \left.\quad-2 f_{1}^{-2} f_{2}^{-2} \Psi_{3 d}\left(f_{1}, f_{2}\right)\right] .
\end{aligned}
$$

The correlation functions $\Psi_{1 d}\left(f_{1}, f_{2}\right)$ and $\Psi_{3 d}\left(f_{1}, f_{2}\right)$ are defined by (24) and (25)-(28), respectively.

For measurements with geometry-free combination (5), the average correction is

$$
\begin{aligned}
\langle & \left.\Delta I_{d}^{(2)}\left(f_{1}, f_{2}\right)\right\rangle \\
& =\left\langle\Delta I_{3 d}^{(2)}\left(f_{1}, f_{2}\right)\right\rangle \\
& =\frac{40.3\left[\bar{I}_{3 d}\left(f_{1}\right)\left(f_{2} / f_{1}\right)^{2}-\bar{I}_{3 d}\left(f_{2}\right)\left(f_{1} / f_{2}\right)^{2}\right]}{\left(f_{1}^{2}-f_{2}^{2}\right)} .
\end{aligned}
$$

Variance of this correction is

$$
\begin{aligned}
\left\langle\left(\Delta \widetilde{I}_{d}^{(2)}\left(f_{1}, f_{2}\right)\right)^{2}\right\rangle= & \left\langle\left(\Delta \widetilde{I}_{1 d}^{(2)}\left(f_{1}, f_{2}\right)\right)^{2}\right\rangle \\
& +\left\langle\left(\Delta \widetilde{I}_{3 d}^{(2)}\left(f_{1}, f_{2}\right)\right)^{2}\right\rangle,
\end{aligned}
$$

where

$$
\begin{aligned}
\left\langle\left(\Delta \widetilde{I}_{1 d}^{(2)}\left(f_{1}, f_{2}\right)\right)^{2}\right\rangle= & \frac{f_{1}^{2} f_{1}^{2}}{\left(f_{1}^{2}-f_{1}^{2}\right)^{2}} \\
& \cdot\left[\left(\frac{f_{1}}{f_{2}}\right)^{-2} \Psi_{1 d}\left(f_{1}, f_{1}\right)+\left(\frac{f_{1}}{f_{2}}\right)^{2}\right. \\
& \left.\cdot \Psi_{1 d}\left(f_{2}, f_{2}\right)-2 \Psi_{1 d}\left(f_{1}, f_{2}\right)\right]
\end{aligned}
$$

is the first-order correction variance and

$$
\begin{aligned}
\left\langle\left(\Delta \widetilde{I}_{3 d}^{(2)}\left(f_{1}, f_{2}\right)\right)^{2}\right\rangle= & \left(\frac{40.3}{f_{1}^{2}-f_{2}^{2}}\right)^{2} \\
& \cdot\left[\left(\frac{f_{1}}{f_{2}}\right)^{-4} \Psi_{3 d}\left(f_{1}, f_{1}\right)+\left(\frac{f_{1}}{f_{2}}\right)^{4}\right. \\
& \left.\cdot \Psi_{3 d}\left(f_{2}, f_{2}\right)-2 \Psi_{3 d}\left(f_{1}, f_{2}\right)\right]
\end{aligned}
$$

is the third-order correction variance. 


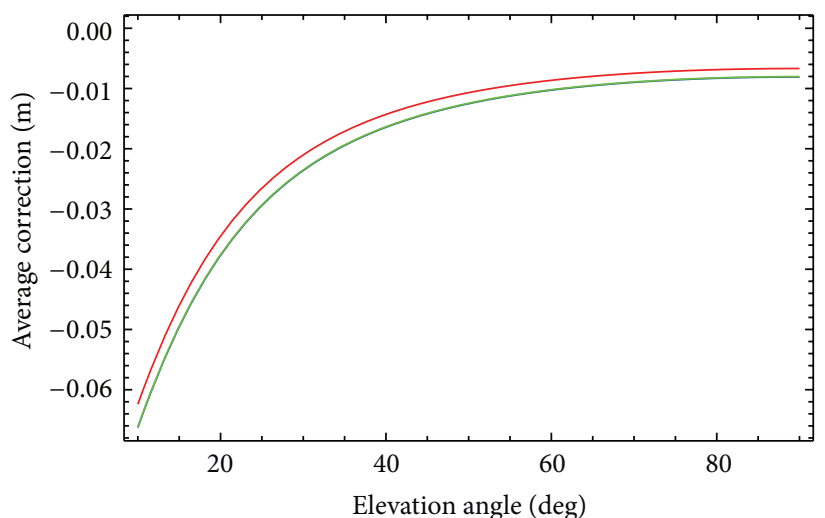

(a)

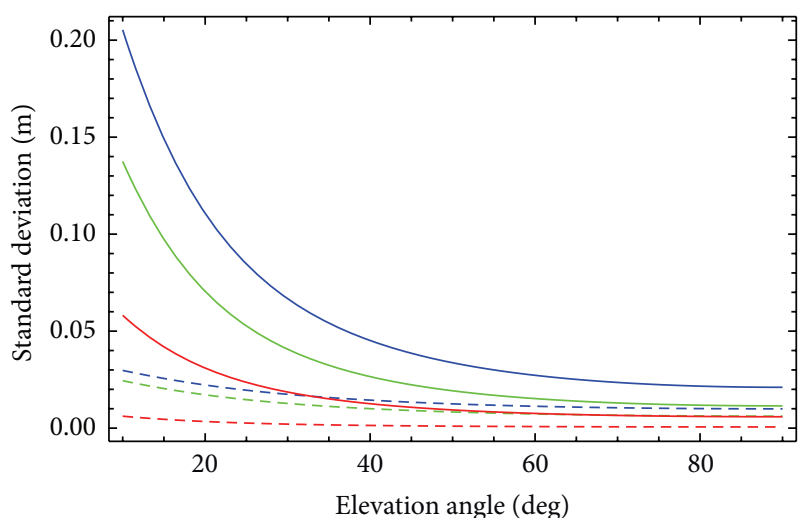

(b)

FIGURE 1: Elevation angle dependence of average (a) and standard deviations (b) of corrections of first (dashed line) and third (solid line) orders for ionosphere-free combination (4) at inner sizes of $50 \mathrm{~m}$ (blue line), $200 \mathrm{~m}$ (green line), and $1000 \mathrm{~m}$ (red line).

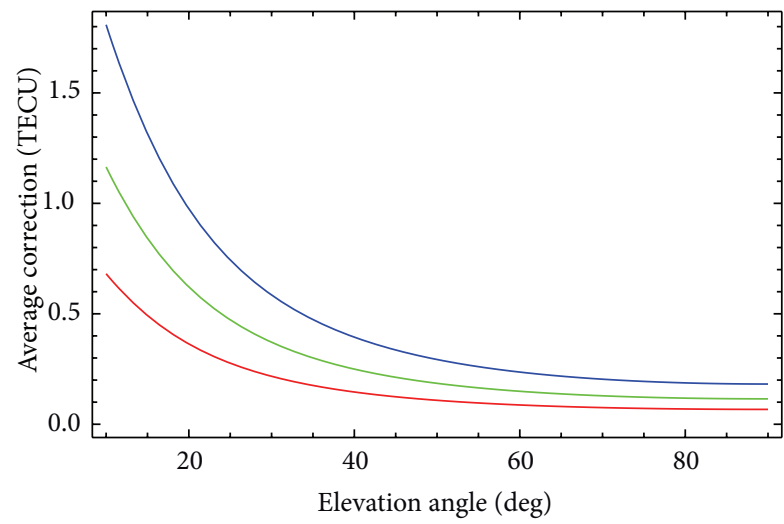

(a)

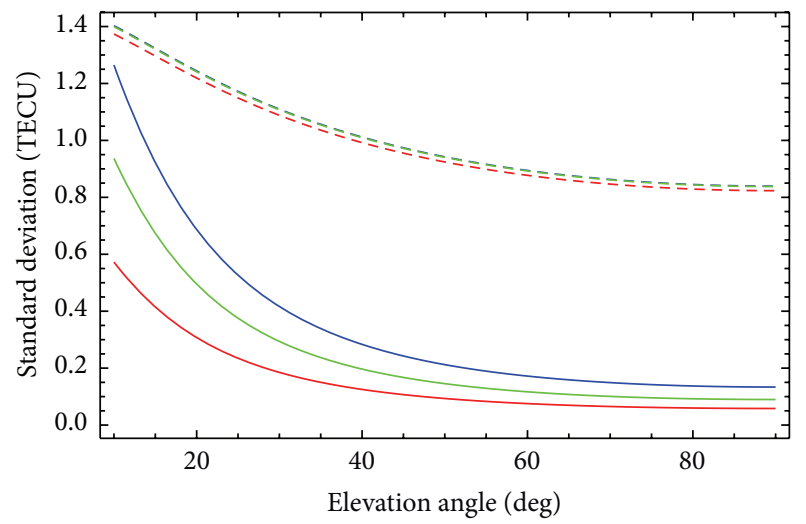

(b)

FIGURE 2: The same as in Figure 1 for geometry-free combination (5).

To assess probable errors in dual-frequency measurements, we have performed numerical simulation with (35)(42). For the model of background ionospheric layer we took the Chapman layer

$$
\bar{N}(z)=\frac{f_{c}^{2}}{80.6} \exp \{0.5[1-\zeta(z)-\exp (\zeta(z))]\},
$$

where $\zeta(z)=\left(\sqrt{z^{2}+2 z R_{e} \sin \alpha+R_{e}^{2}}-h_{m}-R_{e}\right) / H$ and $f_{c}=15 \times 10^{6} \mathrm{~Hz}$ is the layer critical frequency; $R_{e}=$ $6370 \mathrm{~km}$ is the Earth's radius and $H=70 \mathrm{~km}$ is the characteristic scale of the layer; $h_{m}=320 \mathrm{~km}$ is the height of its maximum. Such parameters corresponded to a vertical electron content of 81 TECU $\left(1\right.$ TECU $\left.=10^{16} \mathrm{~m}^{2}\right)$. Random ionospheric irregularities were specified by spectrum (21). Yet we took values for the outer scale $L_{0}=20 \mathrm{~km}$, for the index $p=11 / 3$, and for the coordinate dependence of rms deviation of electron density fluctuation $\sigma_{N}(\mathbf{r})$ we assumed the proportionality to the background electron density $\bar{N}(\mathbf{r})$ : $\sigma_{N}(\mathbf{r})=\sigma_{0} \bar{N}(\mathbf{r})$. In the calculations, we set $\sigma_{0}=0.1$, and for radiation frequencies we took GPS frequencies $f_{1}=$ $1572.42 \times 10^{6} \mathrm{~Hz}, f_{2}=1227.6 \times 10^{6} \mathrm{~Hz}$.
Figures 1 and 2 illustrate elevation angle dependence of average (a) and standard deviations (b) of corrections of first (dashed line) and third (solid line) orders at inner sizes of $50 \mathrm{~m}$ (blue line), $200 \mathrm{~m}$ (green line), and $1000 \mathrm{~m}$ (red line), using the ionosphere-free (Figure 1) and geometry-free (Figure 2) combinations.

A characteristic property of absolute average corrections and standard deviations is their increase with decreasing elevation angle (see Figures 1 and 2) associated with the wavepath extension in the inhomogeneous layer. What stands out here is (see Figure 1(a)) the weak dependence of the average correction in ionosphere-free combination (4) on the inner scale $l_{m}$. This is due to the fact that the greatest contribution to this correction, as is clear from (23), is made by the irregularities located at a distance $z^{\prime}$ where $\kappa_{m}^{2}\left(z_{t}-\right.$ $\left.z^{\prime}\right) / k \gg 1$. In this area, the dependence of the integral in (23) on $\kappa_{m}=2 \pi / l_{m}$ is offset by the factor before the integral. Figure 1(b) shows that the standard deviation of the thirdorder correction (solid line) in $D$ measurements can exceed the standard deviation of the first-order correction (dashed line). The weak dependence of the standard deviation of the first-order (dotted lines in Figure 2(b)) on the inner scale is 
not associated with diffraction effects, as in the case of the average correction in Figure 1(a). Here we deal with phase variance that, as is known [32], is generally determined by large-scale irregularities and slightly depends on the highfrequency part of irregularity spectrum which is associated with the inner scale $l_{m}$. The results of Figure 2 suggest that a probable error in TEC measurements may reach 1 TECU. Third-order corrections increase with decreasing inner sizes, and, as their averages (Figure 2(a)) are other than zero, they cannot be eliminated after time averaging. This, of course, must be taken into account in studying features of ionospheric disturbances of the same order via GNSS. The results of Figure 1 also suggest probable errors in $D$ measurements to be of order of several centimeters, which is comparable with effects measured with GNSS in atmosphere [7].

In [23], numerical simulation was used to explore the possibility of improving the accuracy of GNSS measurements with increasing number of frequencies. It emerged that when the inner scale exceeds the Fresnel radius, the transition from dual-frequency to triple-frequency measurements reduces the ionospheric correction. However, if the Fresnel radius exceeds the inner scale, such a transition decreases only the average correction. The correction variance in this case only rises. Let us, therefore, consider possibilities of improving the accuracy of GNSS measurements with the aid of spatial processing.

\section{Eliminating GNSS Measurement Errors via Fresnel Inversion}

As is well known, with the Green function at a given wave field on the plane where the wave leaves the inhomogeneous medium, it is possible to find the field on another, distant plane. When the medium between these planes is homogeneous, we can take the field of point source in free space as the Green function. This Kirchhoff approximation is widely used for analyzing wave propagation behind the slightly inhomogeneous layer. Yet the wave field where the wave leaves this layer can be described using the geometrical optics approximation. The Kirchhoff formula allows us to calculate this field in the distant region exhibiting various diffraction effects, caustics, and strong fluctuations for which the geometrical optics approximation does not work. Since the small-angle approximation is applicable to large-scale irregularities, the Kirchhoff formula takes the form of Fresnel transform [3]:

$$
\begin{aligned}
U(\boldsymbol{\rho}, z) \approx & -\frac{i k}{2 \pi} \int_{S^{\prime}} U\left(\boldsymbol{\rho}^{\prime}, z_{b}\right) \\
& \cdot \exp \left\{i k \frac{\left(\boldsymbol{\rho}-\boldsymbol{\rho}^{\prime}\right)^{2}}{2\left(z_{t}-z_{b}\right)}+i k\left(z_{t}-z_{b}\right)\right\} d^{2} \rho^{\prime} .
\end{aligned}
$$

When the inhomogeneous layer is sufficiently thin, it modulates only the transmitted wave phase $U\left(\boldsymbol{\rho}^{\prime}, z_{b}\right)=$ $U_{0}\left(z_{b}\right) \exp \left\{i k \varphi_{b}\left(\rho^{\prime}, z_{b}\right)\right\}$ and from (44) we can obtain the phase screen model which has been used for analyzing various problems of wave propagation in a randomly inhomogeneous ionosphere.

On the other hand, inversion of Fresnel transform (44) (Fresnel inversion) allows us, by measuring the field on the plane $z=z_{t}$, to find the field on the plane $z=z_{b}$ nearby the irregularity where diffraction effects are absent and thus increases the accuracy of dual-frequency combinations (4) and (5) in determining $D$ and $I$. Besides reduction of diffraction effects, ray path variations are low and consequently the third-order error is small nearby the irregularity. Let us analyze the action of the inverse operator in the form of inverse Fresnel transform $U\left(\boldsymbol{\rho}, \boldsymbol{\rho}_{0}\right)$ :

$$
\begin{aligned}
\widehat{U}\left(\boldsymbol{\rho}^{*}, z_{b}\right)= & \widehat{L}_{b}\left[U\left(\boldsymbol{\rho}, z_{t}\right)\right]=\frac{i k}{2 \pi\left(z_{t}-z_{b}\right)} \\
& \cdot \int_{-\infty}^{\infty} d^{2} \rho U\left(\boldsymbol{\rho}, z_{t}\right) \exp \left\{-i k \frac{\left(\boldsymbol{\rho}^{*}-\boldsymbol{\rho}\right)^{2}}{2\left(z_{t}-z_{b}\right)}\right\} .
\end{aligned}
$$

Substituting second Rytov approximation (7)-(11) in (45) yields (for details see [28]) formulas analogous to the above ones. When the virtual screen plane $z=z_{b}$ is in a homogeneous medium between an irregularity and an observer, it is the natural result of using the Kirchhoff formula in determining the field on the plane $z=z_{b}$ from values of this field on the plane $z=z_{t}$. However, formally we may apply transform (45) at any position of the virtual screen. This is also suggested by the results of using the double weighted Fourier transform to study wave propagation through a distant inhomogeneous region [33].

Figure 3 presents the results of calculation of the average correction (a) and the standard deviation (b) of first- and third-order corrections in determining the pseudo distance $D$ from ionosphere-free combination (4) after Fresnel processing (45) of GNSS measurements. These results are represented as virtual screen position functions at $l_{m}=50 \mathrm{~m}$ for elevation angles of $90^{\circ}$ (blue line), $50^{\circ}$ (green line), and $20^{\circ}$ (red line). After spatial processing (45), the condition of validity of geometrical optics approximation (16) takes the form

$$
l_{m}^{2} \gg r_{\mathrm{Fr} b}^{2} \equiv \frac{\left(z_{b}-z^{\prime}\right)}{k} .
$$

As is seen from (46), bringing the virtual screen nearer to the irregularity $\left(z_{b} \rightarrow z^{\prime}\right)$ leads to the elimination of diffraction effects. Note that $z_{b}=0$ corresponds to the above case of absence of spatial processing. Figure 3 shows that the absolute average corrections (see Figure 3(a)) and standard deviations (see Figure 3(b)) decrease with increasing distance $z_{b}$ between the observer and the screen and have minimums at the defined $z_{b}=z_{b \text { min. }}$. These minimums for a narrow region with irregularities are close to zero. However, in the case of large extent of the region with irregularities, condition (46) works only for some irregularities and error values in $z_{b}=z_{b \min }$ increase. According to the analysis, these minimums are located at distances corresponding roughly to the points of intersection of the observer-satellite line and 


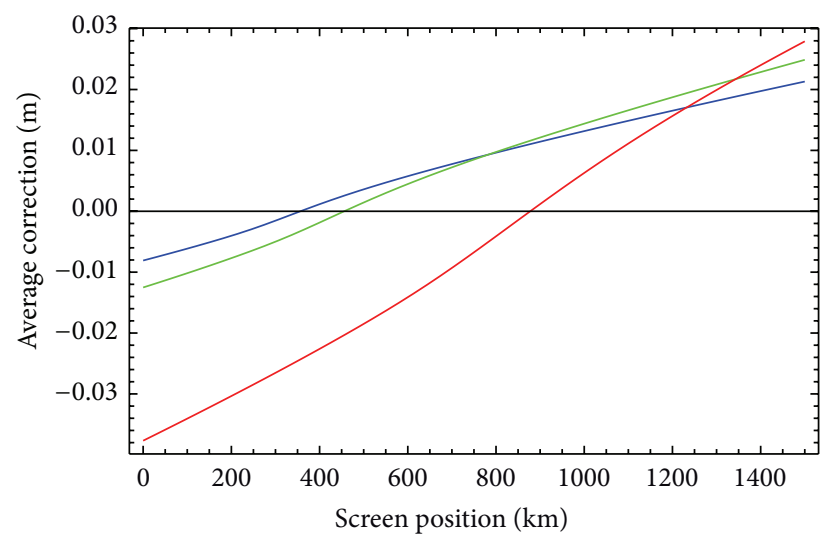

(a)

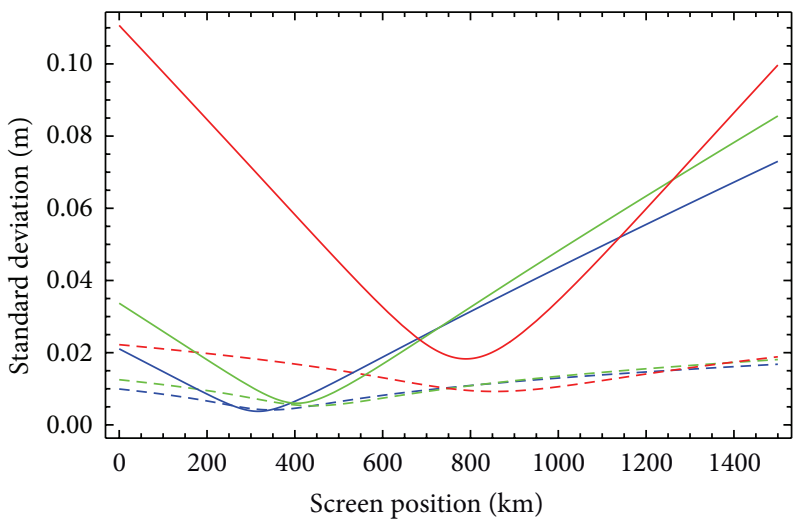

(b)

FiguRE 3: Virtual screen position dependencies of the average (a) and standard deviation (b) of corrections of first (dashed line) and third (solid line) orders for ionosphere-free combination (4) at the inner scale of $50 \mathrm{~m}$ and elevation angles of $90^{\circ}$ (blue line), $50^{\circ}$ (green line), and $20^{\circ}$ (red line).

the layer maximum which for our model of irregularities corresponds to the $\sigma_{N}(\mathbf{r})$ maximum. With this position of the virtual screen, the error is minimal because condition (46) is fulfilled for the most part of the inhomogeneous layer. From the well-known relation between wave amplitude fluctuations and diffraction effects [32] follows an analogous dependence of the amplitude scintillation index on the screen position after spatial processing (45).

Figure 4 for the L1 frequency $\left(f_{1}=1572.42 \times 10^{6} \mathrm{~Hz}\right)$ with the same parameters presents calculations of the amplitude scintillation index $\left\{\left\langle\left|U\left(\boldsymbol{\rho}, \boldsymbol{\rho}_{0}\right)\right|^{4}\right\rangle-\left\langle\left|U\left(\boldsymbol{\rho}, \boldsymbol{\rho}_{0}\right)\right|^{2}\right\rangle^{2}\right\} /$ $\left\langle\left|U\left(\rho, \rho_{0}\right)\right|^{2}\right\rangle^{2}$ of the wave processed by the algorithm of Fresnel inversion (45). This figure implies that processing (45) reduces the scintillation index and the minimum of amplitude fluctuations is very close to the minimum of GNSS measurement errors.

In Figure 5 are the results of the simulation for dualfrequency TEC measurements with geometry-free combination (5). Referring to Figure 5(a), in this case the Fresnel inversion can also reduce the average correction of TEC measurements through the proper choice of distance to the virtual screen $z_{b}$. However, the standard deviation of the first-order correction (dashed lines in Figure 5(b)) varies slightly during spatial processing. This is attributed to the said (Figure 2(b)) relationship between the variance of the firstorder correction and the variance of the first-approximation phase which weakly depends on diffraction effects [32]. However, the standard deviation of the third-order correction (solid lines in Figure 5(b)) in optimum Fresnel processing (at $z_{b}=z_{b \min }$ ) reduces due to elimination of the diffraction effects and decrease in the ray bending.

\section{Conclusion}

In this study, ionospheric errors of GNSS remote sensing using both ionosphere-free and geometry-free combinations were analyzed. In the former case, we demonstrated the possibility of reducing residual ionospheric errors in

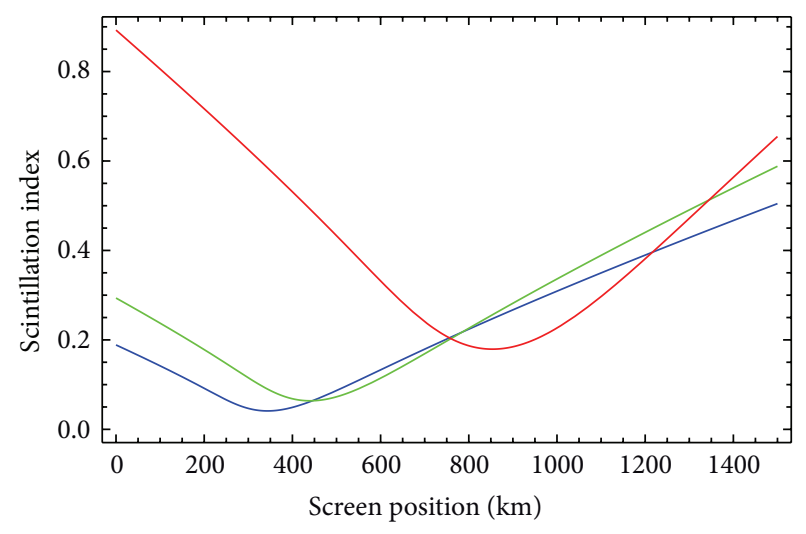

FIGURE 4: Virtual screen position dependencies of the scintillation index for the frequency $\mathrm{L} 1\left(f_{1}=1572.42 \times 10^{6} \mathrm{~Hz}\right)$ for the same elevation angles as in Figure 3.

ionosphere-free combination by Fresnel inversion. The inversion parameter, the distance to the virtual screen, may be selected from the minimum of amplitude fluctuations. This suggests the possibility of improving the accuracy of GNSS remote sensing in meteorology.

In investigations into ionospheric disturbances through geometry-free combinations, the Fresnel inversion eliminates only the third-order correction. To eliminate the random TEC component which, like the measured average TEC, is the first-order correction, we should use temporal filtering (averaging). However, measurements of this random TEC component allow us to examine the fine structure of ionospheric plasma via GNSS.

The Fresnel spatial processing requires constructing a phase GNSS array of sufficiently large sizes. Despite the difficulty in constructing this array, it seems necessary to develop the array, taking into account feasibility of using it in many fields of research on the troposphere, ionosphere, earthquakes, and volcanic activity. 


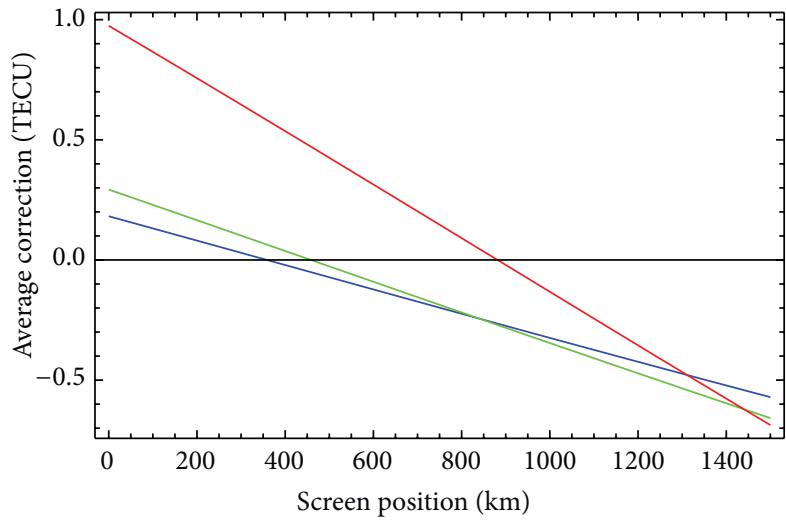

(a)

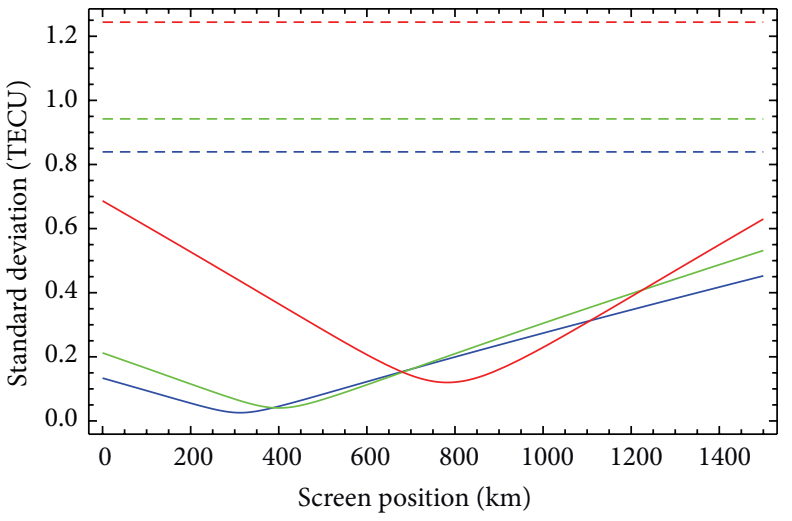

(b)

Figure 5: The same as in Figure 3 for geometry-free combination (5).

\section{Conflict of Interests}

The author declares that there is no conflict of interests regarding the publication of this paper.

\section{Acknowledgments}

The author is very grateful to O. A. Kulish for her assistance in preparing the English version of the paper. This study was supported by the grant from the Russian Scientific Foundation (Project no. 14-37-00027).

\section{References}

[1] S. Kedar, G. A. Hajj, B. D. Wilson, and M. B. Heflin, "The effect of the second order GPS ionospheric correction on receiver positions," Geophysical Research Letters, vol. 30, no. 16, 2003.

[2] E. R. Kursinski, G. A. Hajj, J. T. Schofield, R. P. Linfield, and K. R. Hardy, "Observing Earth's atmosphere with radio occultation measurements using the global positioning system," Journal of Geophysical Research D: Atmospheres, vol. 102, no. 19, pp. 2342923465, 1997.

[3] M. D. Moïtensen and P. Høeg, "Inversion of GPS occultation measurements using Fresnel diffraction theory," Geophysical Research Letters, vol. 25, no. 13, pp. 2441-2444, 1998.

[4] R. E. Schaal and A. P. C. Larocca, "A methodology for monitoring vertical dynamic sub-centimeter displacements with GPS," GPS Solutions, vol. 5, no. 3, pp. 15-18, 2002.

[5] H. C. Baker, A. H. Dodson, N. T. Penna, M. Higgins, and D. Offiler, "Ground-based GPS water vapour estimation: potential for meteorological forecasting," Journal of Atmospheric and Solar-Terrestrial Physics, vol. 63, no. 12, pp. 1305-1314, 2001.

[6] V. Janssen, "GPS volcano deformation monitoring (GPS on the Web)," GPS Solutions, vol. 6, pp. 128-130, 2002.

[7] S. Jin, E. Cardellach, and F. Xie, GNSS Remote Sensing. Theory, Methods and Applications, Springer, Dordrecht, The Netherlands, 2014.

[8] V. Hoyle, S. Skone, and S. Shrestha, "The use of the global positioning system to derive atmospheric water vapor distribution for environmental applications," Environmental Informatics Archives, vol. 1, pp. 512-523, 2003.
[9] M. S. Grewal, L. R. Weill, and A. P. Andrews, Global Positioning Systems, Inertial Navigation, and Integration, Wiley, New York, NY, USA, 2007.

[10] G. Xu, GPS Theory, Algorithms and Applications, Springer, New York, NY, USA, 2nd edition, 2007.

[11] J. B. Tsui, Fundamentals of Global Positioning System Receivers: A Software Approach, John Wiley \& Sons, Hoboken, NJ, USA, 2nd edition, 2005.

[12] P. J. G. Teunissen, "Theory of integer equivariant estimation with application to GNSS," Journal of Geodesy, vol. 77, no. 7-8, pp. 402-410, 2003.

[13] S. Bassiri and G. A. Hajj, "Higher-order ionospheric effects on the global positioning system observables and means of modeling them," Manuscripta Geodaetica, vol. 18, pp. 280-289, 1993.

[14] M. Fritsche, R. Dietrich, C. Knöfel et al., "Impact of higherorder ionospheric terms on GPS estimates," Geophysical Research Letters, vol. 32, no. 23, 2005.

[15] M. M. Hoque and N. Jakowski, "Estimate of higher order ionospheric errors in GNSS positioning," Radio Science, vol. 43, no. 5, Article ID RS5008, 2008.

[16] G. Beutler, E. Brockmann, S. Fankhauser et al., Bernese GPS Software Version 4.0, University of Berne, Bern, Switzerland, 1996.

[17] J. A. Klobuchar and J. M. Kunches, "Comparative range delay and variability of the earth's troposphere and the ionosphere," GPS Solutions, vol. 7, no. 1, pp. 55-58, 2003.

[18] M. Elsobeiey and A. El-Rabbany, "On modelling of secondorder ionospheric delay for GPS precise point positioning," The Journal of Navigation, vol. 65, no. 1, pp. 59-72, 2012.

[19] M. Hernández-Pajares, J. M. Juan, J. Sanz, and R. Orús, "Secondorder ionospheric term in GPS: implementation and impact on geodetic estimates," Journal of Geophysical Research: Solid Earth, vol. 112, Article ID B08417, 2007.

[20] H. Munekane, "A semi-analytical estimation of the effect of second-order ionospheric correction on the GPS positioning," Geophysical Journal International, vol. 163, no. 1, pp. 10-17, 2005.

[21] K. Palamartchouk, "Apparent geocenter oscillations in Global Navigation Satellite Systems solutions caused by the ionospheric effect of second order," Journal of Geophysical Research B: Solid Earth, vol. 115, no. 3, Article ID B03415, 2010. 
[22] M. V. Tinin and E. V. Konetskaya, "Influence of geomagnetic field on the ionospheric error of satellite navigation systems," Geomagnetism and Aeronomy, vol. 53, pp. 741-749, 2013.

[23] B. C. Kim and M. V. Tinin, "Potentialities of multifrequency ionospheric correction in Global Navigation Satellite Systems," Journal of Geodesy, vol. 85, no. 3, pp. 159-169, 2011.

[24] B.-C. Kim and M. V. Tinin, "Effect of ionospheric irregularities on accuracy of dual-frequency GPS systems," Geomagnetism and Aeronomy, vol. 47, no. 2, pp. 238-243, 2007.

[25] K. C. Yeh and C. H. Liu, "Radio-wave scintillations in the ionosphere," Proceedings of the IEEE, vol. 70, no. 4, pp. 324-360, 1982.

[26] B. N. Gershman, L. M. Erukhimov, and Y. Y. Yashin, Wave Phenomena in the Ionosphere and Cosmic Plasma, Nauka, Moscow, Russia, 1984, (Russian).

[27] A. D. Wheelon, Electromagnetic Scintillation II. Weak Scattering, Cambridge University Press, Cambridge, UK, 2001.

[28] M. V. Tinin, "Eliminating diffraction effects during multifrequency correction in global navigation satellite systems," Journal of Geodesy, vol. 89, 2015.

[29] E. L. Afraimovich, N. P. Perevalova, and S. V. Voyeikov, "Traveling wave packets of total electron content disturbances as deduced from global GPS network data," Journal of Atmospheric and Solar-Terrestrial Physics, vol. 65, no. 11-13, pp. 1245-1262, 2003.

[30] V. I. Tatarskii, The Effect of a Turbulent Atmosphere on Wave Propagation, National Technical Information Service, Springfield, Va USA, 1971.

[31] B. C. Kim and M. V. Tinin, “The second-order Rytov approximation and residual error in dual-frequency satellite navigation systems," Waves in Random and Complex Media, vol. 19, no. 2, pp. 284-304, 2009.

[32] S. M. Rytov, Y. A. Kravtsov, and V. I. Tatarskii, Introduction to Statistical Radiophysics, Vol 4. Wave Propagation through Random Media, Springer, New York, NY, USA, 1989.

[33] M. V. Tinin, "Integral representation of the field of the wave propagating in a medium with large-scale irregularities," Radiophysics and Quantum Electronics, vol. 55, no. 6, pp. 391-398, 2012. 

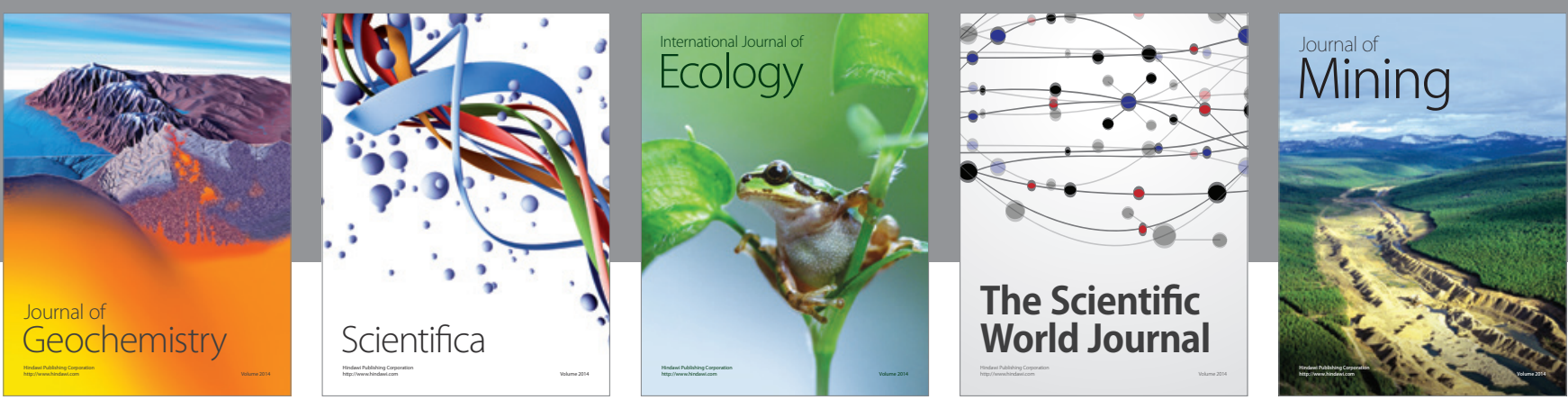

The Scientific World Journal
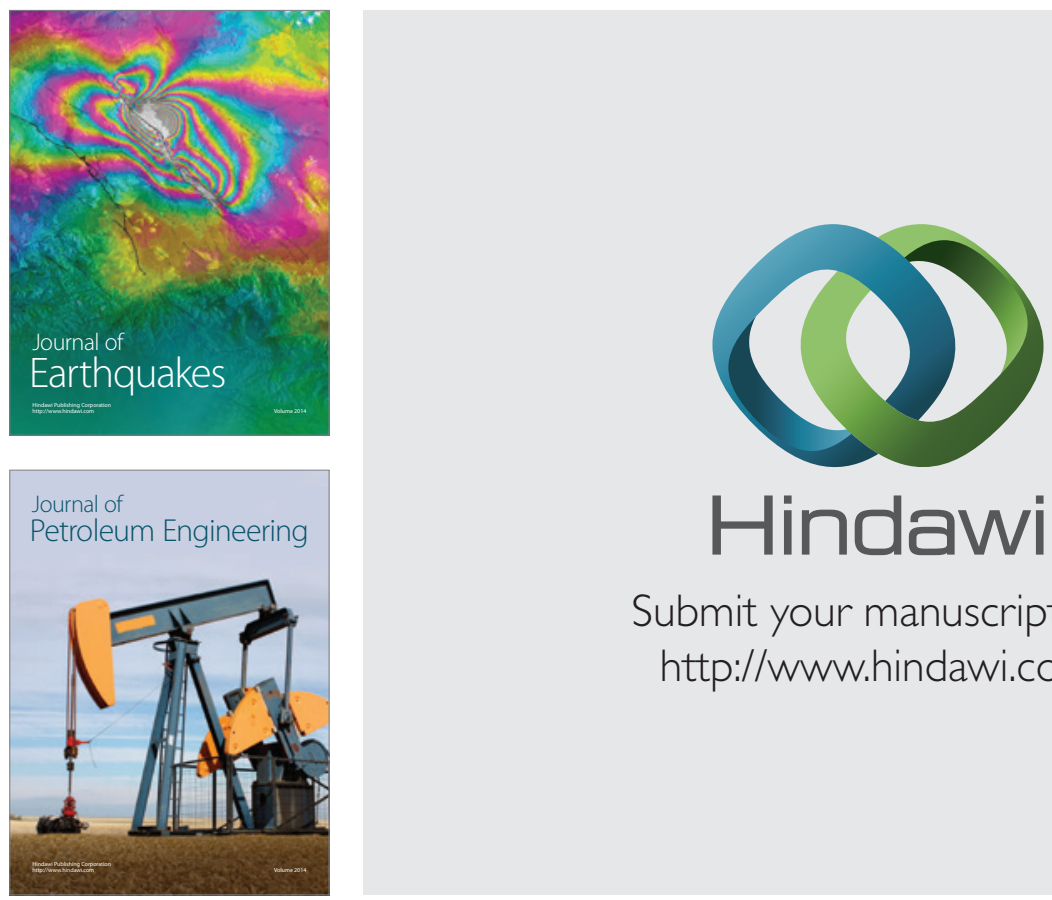

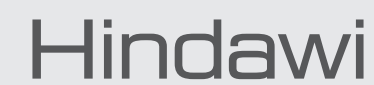

Submit your manuscripts at

http://www.hindawi.com
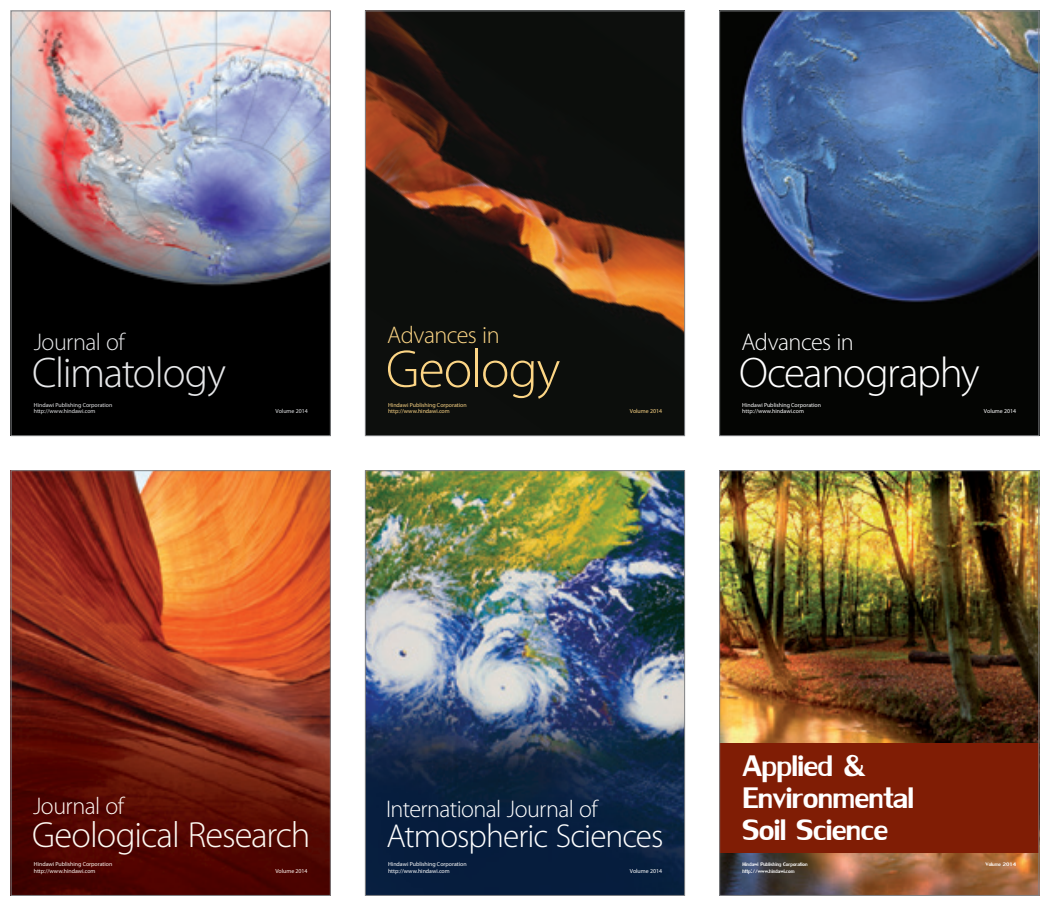
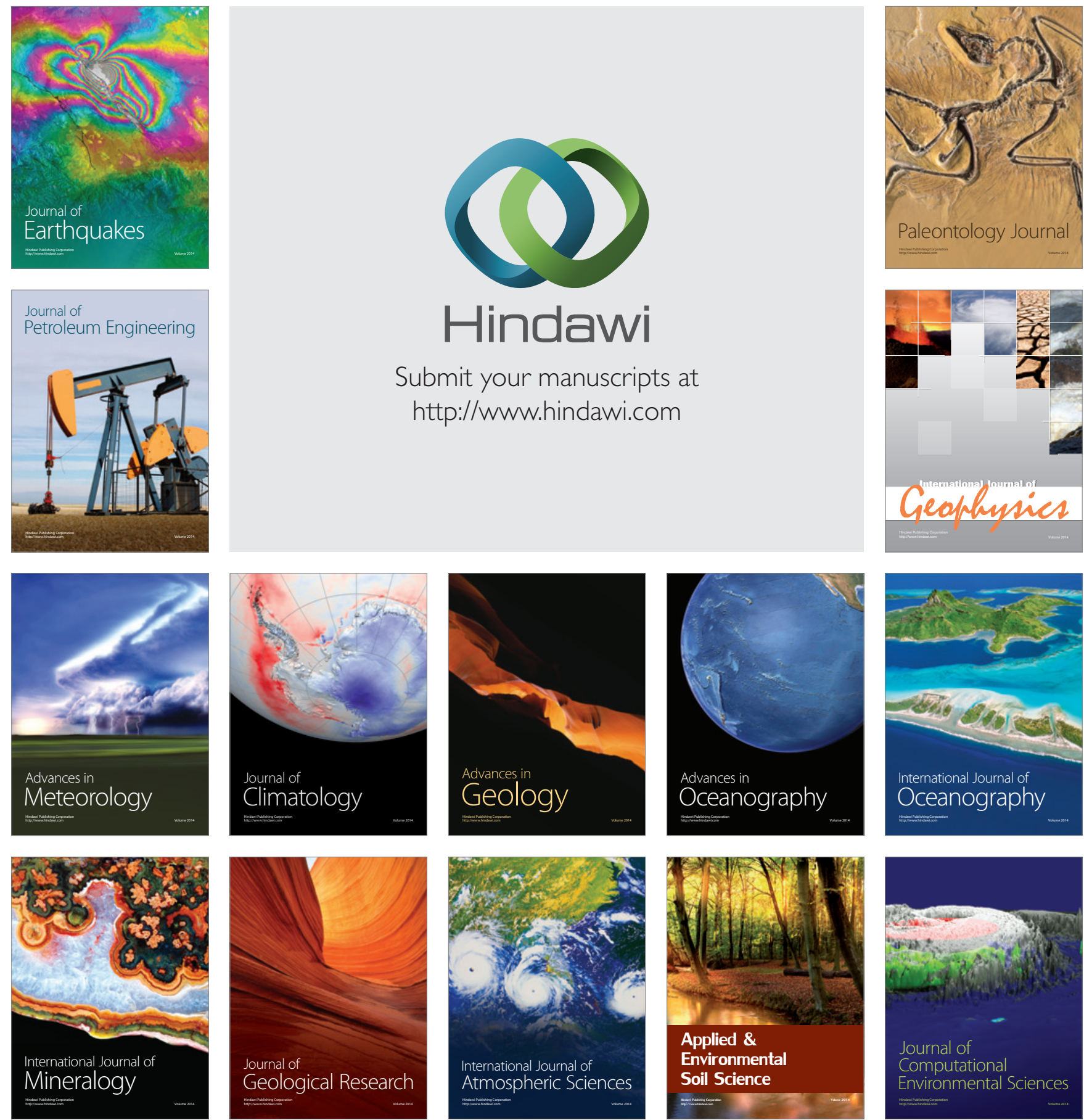